\title{
Varieties of coarse spaces
}

\section{Igor Protasov}

\begin{abstract}
A class $\mathfrak{M}$ of coarse spaces is called a variety if $\mathfrak{M}$ is closed under formation of subspaces, coarse images and products. We classify the varieties of coarse spaces and, in particular, show that if a variety $\mathfrak{M}$ contains an unbounded metric space then $\mathfrak{M}$ is the variety of all coarse spaces.
\end{abstract}

MSC: 54E35, 08B85.

Keywords: coarse structure,coarse space, ballean, varieties of coarse spaces.

\section{Introduction}

Following [9], we say that a family $\mathcal{E}$ of subsets of $X \times X$ is a coarse structure on a set $X$ if

- each $\varepsilon \in \mathcal{E}$ contains the diagonal $\triangle_{X}, \triangle_{X}=\{(x, x): x \in X\}$;

- if $\varepsilon, \delta \in \mathcal{E}$ then $\varepsilon \circ \delta \in \mathcal{E}$ and $\varepsilon^{-1} \in \mathcal{E}$ where $\varepsilon \circ \delta=\{(x, y): \exists z((x, z) \in \varepsilon,(z, y) \in \delta)\}$, $\varepsilon^{-1}=\{(y, x):(x, y) \in \varepsilon\}$

- if $\varepsilon \in \mathcal{E}$ and $\triangle_{X} \subseteq \varepsilon^{\prime} \subseteq \varepsilon$ then $\varepsilon^{\prime} \in \mathcal{E}$.

Each $\varepsilon \in \mathcal{E}$ is called an entourage of the diagonal. A subset $\mathcal{E}^{\prime} \subseteq \mathcal{E}$ is called a base for $\mathcal{E}$ if, for every $\varepsilon \in \mathcal{E}$ there exists $\varepsilon^{\prime} \in \mathcal{E}^{\prime}$ such that $\varepsilon \subseteq \varepsilon^{\prime}$.

The pair $(X, \mathcal{E})$ is called a coarse space. For $x \in X$ and $\varepsilon \in \mathcal{E}$, we denote $B(x, \varepsilon)=$ $\{y \in X:(x, y) \in \varepsilon\}$ and say that $B(x, \varepsilon)$ is a ball of radius $\varepsilon$ around $x$. We note that a coarse space can be considered as an asymptotic counterpart of a uniform topological space and could be defined in terms of balls, see [6], [8]. In this case a coarse space is called a ballean.

A coarse space $(X, \mathcal{E})$ is called connected if, for any $x, y \in X$, there exists $\varepsilon \in \mathcal{E}$ such that $y \in B(x, \varepsilon)$. A subset $Y$ of $X$ is called bounded if there exist $x \in X$ and $\varepsilon \in \mathcal{E}$ such that $Y \subseteq B(x, \varepsilon)$. The coarse structure $\mathcal{E}=\left\{\varepsilon \in X \times X: \triangle_{X} \subseteq \varepsilon\right\}$ is the unique coarse structure such that $(X, \mathcal{E})$ is connected and bounded.

In what follows, all coarse spaces under consideration are supposed to be connected.

Given a coarse space $(X, \mathcal{E})$, each subset $Y \subseteq X$ has the natural coarse structure $\left.\mathcal{E}\right|_{Y}=\{\varepsilon \cap(Y \times Y): \varepsilon \in \mathcal{E}\},\left(Y,\left.\mathcal{E}\right|_{Y}\right)$ is called a subspace of $(X, \mathcal{E})$. A subset $Y$ of $X$ is called large (or coarsely dense) if there exists $\varepsilon \in \mathcal{E}$ such that $X=B(Y, \varepsilon)$ where $B(Y, \varepsilon)=\cup_{y \in Y} B(Y, \varepsilon)$. 
Let $(X, \mathcal{E}),\left(X^{\prime}, \mathcal{E}^{\prime}\right)$ be coarse spaces. A mapping $f: X \longrightarrow X^{\prime}$ is called coarse (or bornologous in terminology of [9]) if, for every $\varepsilon \in \mathcal{E}$ there exists $\varepsilon^{\prime} \in \mathcal{E}^{\prime}$ such that, for every $x \in X$, we have $f(B(x, \varepsilon)) \subseteq\left(B\left(f(x), \varepsilon^{\prime}\right)\right)$. If $f$ is surjective and coarse then $\left(X^{\prime}, \mathcal{E}^{\prime}\right)$ is called a coarse image of $(X, \mathcal{E})$. If $f$ is a bijection such that $f$ and $f^{-1}$ are coarse mappings then $f$ is called an asymorphism. The coarse spaces $(X, \mathcal{E}),\left(X^{\prime}, \mathcal{E}^{\prime}\right)$ are called coarsely equivalent if there exist large subsets $Y \subseteq X, Y^{\prime} \subseteq X$ such that $\left(Y,\left.\mathcal{E}\right|_{Y}\right)$ and $\left(Y^{\prime},\left.\mathcal{E}^{\prime}\right|_{Y^{\prime}}\right)$ are asymorphic.

To conclude the coarse vocabulary, we take a family $\left\{\left(X_{\alpha}, \mathcal{E}_{\alpha}\right): \alpha<\kappa\right\}$ of coarse spaces and define the product $P_{\alpha<\kappa}\left(X_{\alpha}, \mathcal{E}_{\alpha}\right)$ as the set $P_{\alpha<\kappa} X_{\alpha}$ endowed with the coarse structure with the base $P_{\alpha<\kappa} \mathcal{E}_{\alpha}$. If $\varepsilon_{\alpha} \in \mathcal{E}_{\alpha}, \alpha<\kappa$ and $x, y \in P_{\alpha<\kappa} X_{\alpha}, x=\left(x_{\alpha}\right)_{\alpha<\kappa}$, $y=\left(y_{\alpha}\right)_{\alpha<\kappa}$ then $(x, y) \in\left(\varepsilon_{\alpha}\right)_{\alpha<\kappa}$ if and only if $\left(x_{\alpha}, y_{\alpha}\right) \in \varepsilon_{\alpha}$ for every $\alpha<\kappa$.

Let $\mathfrak{M}$ be a class of coarse spaces closed under asymorphisms. We say that $\mathfrak{M}$ is a variety if $\mathfrak{M}$ is closed under formation of subspaces $(\mathbf{S M} \subseteq \mathfrak{M})$, coarse images $(\mathbf{Q M} \subseteq \mathfrak{M})$ and products $(\mathbf{P} \mathfrak{M} \subseteq \mathfrak{M})$.

For an infinite cardinal $\kappa$, we say that a coarse space $(X, \mathcal{E})$ is $\kappa$-bounded if every subset $Y \subseteq X$ such that $|Y|<\kappa$ is bounded, and denote by $\mathfrak{M}_{\kappa}$ the variety of all $\kappa$-bounded coarse spaces.

We denote by $\mathfrak{M}_{\text {single }}$ and $\mathfrak{M}_{\text {bound }}$ the variety of singletons and the variety of all bounded coarse spaces.

Then we get the chain of varieties

$$
\mathfrak{M}_{\text {single }} \subset \mathfrak{M}_{\text {bound }} \subset \ldots \subset \mathfrak{M}_{\kappa} \subset \ldots \subset \mathfrak{M}_{\omega}
$$

In section 2 , we prove that every variety of coarse spaces lies in this chain and, in section 3 , we discuss some extensions of this result to coarse spaces endowed with additional algebraic structures.

\section{Results}

We recall that a family $\mathcal{I}$ of subsets of a set $X$ is an ideal in the Boolean algebra $\mathcal{P}_{X}$ of all subsets of $X$ if $\mathcal{I}$ is closed under finite unions and subsets. Every ideal $\mathcal{I}$ defines the coarse structure with the base $\left\{\mathcal{E}_{A}: A \in \mathcal{I}\right\}$ where $\mathcal{E}_{A}=(A \times A) \cup \triangle_{X}$, so $B\left(x, \mathcal{E}_{A}\right)=A$ if $x \in A$ and $B\left(x, \mathcal{E}_{A}\right)=\{x\}$ if $x \in X \backslash A$. We denote the obtained coarse space by $(X, \mathcal{I})$. For a cardinal $\kappa,[X]^{<\kappa}$ denotes the ideal $\{Y \subseteq X:|Y|<\kappa\}$. If $(X, \mathcal{E})$ is a coarse space, the family $\mathcal{I}$ of all bounded subsets of $X$ is an ideal. The coarse space $(X, \mathcal{I})$ is called the companion of $(X, \mathcal{E})$.

Let $\mathcal{K}$ be a class of coarse spaces. We say that a coarse space $(X, \mathcal{E})$ is free with respect to $\mathcal{K}$ if, for every $\left(X^{\prime}, \mathcal{E}^{\prime}\right) \in \mathcal{K}$ every mapping $f:(X, \mathcal{E}) \longrightarrow\left(X^{\prime}, \mathcal{E}^{\prime}\right)$ is coarse. For example, $\left(X,[X]^{<\kappa}\right)$ is free with respect to the variety $\mathfrak{M}_{\kappa}$. Since $\left(\kappa,[\kappa]^{<\kappa}\right) \in \mathfrak{M}_{\kappa}$ but $\left(\kappa,[\kappa]^{<\kappa}\right) \notin \mathfrak{M}_{\kappa^{\prime}}$ for each $\kappa^{\prime}>\kappa$, the inclusion $\mathfrak{M}_{\kappa^{\prime}} \subset \mathfrak{M}_{\kappa}$ is strict.

Lemma 1. If a coarse space $(X, \mathcal{E})$ is free with respect to a class $\mathcal{K}$ then $(X, \mathcal{E})$ is free with respect to $\mathbf{S} \mathcal{K}, \mathbf{Q} \mathcal{K}, \mathbf{P} \mathcal{K}$. 
Proof. We verify only the second statement. Let $\left(X^{\prime}, \mathcal{E}^{\prime}\right) \in \mathcal{K},\left(X^{\prime \prime}, \mathcal{E}^{\prime \prime}\right) \in \mathrm{Q} \mathcal{K}$, and $h:\left(X^{\prime}, \mathcal{E}^{\prime}\right) \longrightarrow\left(X^{\prime \prime}, \mathcal{E}^{\prime \prime}\right)$ be a coarse surjective mapping. We take an arbitrary $f: X \longrightarrow X^{\prime \prime}$ and choose $h^{\prime}: X \longrightarrow X^{\prime}$ such that $f=h h^{\prime}$. Since $(X, \mathcal{E})$ is free with respect to $\mathcal{K}, h^{\prime}:(X, \mathcal{E}) \longrightarrow\left(X^{\prime}, \mathcal{E}^{\prime}\right)$ is coarse so $f$ is coarse as the composition of the coarse mappings $h, h^{\prime}$.

Lemma 2. Let $X$ be a set and let $\mathcal{K}$ be a class of coarse spaces, $\mathcal{K} \neq \mathfrak{M}_{\text {single. }}$ Then there exists a coarse structure $\mathcal{E}$ on $X$ such that $(X, \mathcal{E}) \in \mathbf{S P} \mathcal{K}$ and $(X, \mathcal{E})$ is free with respect to $\mathcal{K}$.

Proof. We take a set $S$ of all pairwise non-asymorphic coarse spaces $\left(X^{\prime}, \mathcal{E}^{\prime}\right) \in \mathcal{K}$ such that $\left|X^{\prime}\right| \leq|X|$ and enumerate all possible triplets $\left\{\left(X_{\alpha}, \mathcal{E}_{\alpha}, f_{\alpha}\right): \alpha<\lambda\right\}$ such that $\left(X_{\alpha}, \mathcal{E}_{\alpha}\right) \in S$ and $f_{\alpha}: X \longrightarrow X_{\alpha}$. Then we consider the product $P_{\alpha<\lambda}\left(X_{\alpha}, \mathcal{E}_{\alpha}\right)$ and define $f: X \longrightarrow P_{\alpha<\lambda} X_{\alpha}$ by $f(x)=\left(f_{\alpha}(x)\right)_{\alpha<\lambda}$. Since $\mathcal{K} \neq \mathfrak{M}_{\text {single }}, f$ is injective so we can identify $X$ with $f(X)$ and consider the subspace $(X, \mathcal{E})$ of $P_{\alpha<\lambda}\left(X_{\alpha}, \mathcal{E}_{\alpha}\right)$. Clearly, $(X, \mathcal{E}) \in \mathbf{S P K}$.

To see that $(X, \mathcal{E})$ is free with respect to $\mathcal{K}$, it suffices to verify that, for each $\left(X^{\prime}, \mathcal{E}^{\prime}\right) \in S$, every mapping $h:(X, \mathcal{E}) \longrightarrow\left(X^{\prime}, \mathcal{E}^{\prime}\right)$ is coarse. We take $\beta<\lambda$ such that $\left(X^{\prime}, \mathcal{E}^{\prime}\right)=\left(X_{\beta}, \mathcal{E}_{\beta}\right)$ and $h=f_{\beta}$. Then $f_{\beta}$ is the restriction to $X$ of the projection $\operatorname{pr}_{\beta}: P_{\alpha<\lambda}\left(X_{\alpha}, \mathcal{E}_{\alpha}\right) \longrightarrow\left(X_{\beta}, \mathcal{E}_{\beta}\right)$ so $f_{\beta}$ is coarse.

Theorem 1. For every class $\mathcal{K}$ of coarse spaces, the smallest variety Var $\mathcal{K}$ containing $\mathcal{K}$ is QSPK.

Proof.The inclusion QSP $\mathcal{K} \subseteq \mathcal{K}$ is evident. To prove the inverse inclusion, we suppose that $\mathcal{K} \neq \mathfrak{M}_{\text {single }}$ (this case is evident) and take an arbitrary $\left(X^{\prime}, \mathcal{E}^{\prime}\right) \in \operatorname{Var}(\mathcal{K})$. Then $\left(X^{\prime}, \mathcal{E}^{\prime}\right)$ can be obtained from $\mathcal{K}$ by means of some finite sequence of operations $\mathbf{S}, \mathbf{P}, \mathbf{Q}$. We use Lemma 2 to choose a coarse space $(X, \mathcal{E}) \in \mathbf{S P K},|X|=\left|X^{\prime}\right|$ free with respect to $\mathcal{K}$. By Lemma 1 , any bijection $f:(X, \mathcal{E}) \longrightarrow\left(X^{\prime}, \mathcal{E}^{\prime}\right)$ is coarse so $\left(X^{\prime}, \mathcal{E}^{\prime}\right) \in \mathbf{Q S P} \mathcal{K}$.

Theorem 2. Let $\mathfrak{M}$ be a variety of coarse spaces such that $\mathfrak{M} \neq \mathfrak{M}_{\text {single }}, \mathfrak{M} \neq \mathfrak{M}_{\text {bound }}$. Then there exists a cardinal $\kappa$ such that $\mathfrak{M}=\mathfrak{M}_{\kappa}$.

Proof. Since $\mathfrak{M} \neq \mathfrak{M}_{\text {bound }}$ and $\mathfrak{M} \neq \mathfrak{M}_{\text {single }}$, there exists the minimal cardinal $\kappa$ such that each space from $\mathfrak{M}$ is $\kappa$-bounded so $\mathfrak{M} \subseteq \mathfrak{M}_{\kappa}$.

To verify the inclusion $\mathfrak{M}_{\kappa} \subseteq \mathfrak{M}$, we take a coarse space $(X, \mathcal{E}) \in \mathfrak{M}$ free with respect to $\mathfrak{M}$ and show that $(X, \mathcal{E})$ is free with respect to $\mathfrak{M}_{\kappa}$. We prove that $(X, \mathcal{E})=\left(X,[X]^{<\kappa}\right)$. If $|X|<\kappa$ then $(X, \mathcal{E})$ is bounded and the statement is evident. Assume that $|X| \geq \kappa$ but $(X, \mathcal{E}) \neq\left(X,[X]^{<\kappa}\right)$. Assume that, for every $\varepsilon, \varepsilon=\varepsilon^{-1}$, the set $S_{\varepsilon}=\{x \in X$ : $|B(x, \varepsilon)|>1\}$ is bounded in $(X, \mathcal{E})$. By the choice of $\kappa,\left|S_{\varepsilon}\right|<\kappa$ and $|B(x, \varepsilon)|=1$ for all $x \in X \backslash S_{\varepsilon}$. It follows that $(X, \mathcal{E})=\left(X,[X]^{<\kappa}\right)$. Then there exists $\varepsilon \in \mathcal{E}$ such that the set $S_{\varepsilon}$ is unbounded in $(X, \mathcal{E})$. We choose a maximal by inclusion subset $Y \subset X$ such 
that $B(y, \varepsilon) \cap B\left(y^{\prime}, \varepsilon\right)=\emptyset$ for all distinct $y, y^{\prime} \in Y$. We observe that $Y$ is unbounded so $|Y| \geq \kappa$. We take an arbitrary $x_{0} \in X$ and choose a mapping $f: X \longrightarrow X$ such that $f(y)=x_{0}$ for each $y \in Y$ and $f$ is injective on $X \backslash Y$. Since $(X, \mathcal{E})$ is free with respect to $\mathfrak{M}$, the mapping $f:(X, \mathcal{E}) \longrightarrow(X, \mathcal{E})$ must be coarse. Hence, there exists $\varepsilon^{\prime} \in \mathcal{E}$ such that $f(B(x, \varepsilon)) \subseteq B\left(f(x), \varepsilon^{\prime}\right)$ for each $x \in X$. It follows that $f\left(\cup_{y \in Y} B(y, \varepsilon)\right)$ is bounded in $(X, \mathcal{E})$. We note that $\left|f\left(\cup_{y \in Y} B(y, \varepsilon)\right)\right| \geq \kappa$ so $(X, \mathcal{E})$ contains a bounded subset $Z$ such that $|Z|=\kappa$. Since $(X, \mathcal{E})$ is free with respect to $\mathfrak{M}$, every $\left(X^{\prime}, \varepsilon^{\prime}\right) \in \mathfrak{M}$ is a $\kappa^{+}$bounded and we get a contradiction with the choice of $\kappa$. To conclude the proof, we take an arbitrary $\left(X, \mathcal{E}^{\prime}\right) \in \mathfrak{M}_{\kappa}$ and note that the identity mapping $i d:\left(X,[X]^{<\kappa}\right) \longrightarrow\left(X, \mathcal{E}^{\prime}\right)$ is coarse so $\left(X, \mathcal{E}^{\prime}\right) \in \mathfrak{M}$.

Remark 1. We note that $\mathfrak{M}_{\text {single }}$ is not closed under coarse equivalence because each bounded coarse space is coarsely equivalent to a singleton. Clearly, $\mathfrak{M}_{\text {bound }}$ is closed under coarsely equivalence. We show that the same is true for every variety $\mathfrak{M}_{\kappa}$. Let $(X, \mathcal{E})$ be a coarse space, $Y$ be a large subset of $(X, \mathcal{E})$. We assume that $\left(Y,\left.\mathcal{E}\right|_{Y}\right) \in \mathfrak{M}_{\kappa}$ but $(X, \mathcal{E}) \notin \mathfrak{M}_{\kappa}$. Then $X$ contains an unbounded subset $Z$ such that $|Z|<\kappa$. We choose $\varepsilon \in \mathcal{E}$ such that $\varepsilon=\varepsilon^{-1}$ and $X=B(Y, \mathcal{E})$. For each $z \in Z$, we pick $y_{z} \in Y$ such that $z \in B\left(y_{z}, \mathcal{E}\right)$. We put $Y^{\prime}=\left\{y_{z} \in Z\right\}$. Since $\left|Y^{\prime}\right|<\kappa, Y^{\prime}$ is bounded in $\left(Y,\left.\mathcal{E}\right|_{Y}\right)$. It follows that $Z$ is bounded in $(X, \mathcal{E})$, a contradiction with the choice of $Z$.

We note also that every variety of coarse spaces is closed under formations of companions. For $\mathfrak{M}_{\text {single }}$ and $\mathfrak{M}_{\text {bound }}$, this is evident. Let $(X, \mathcal{E}) \in \mathfrak{M}_{\kappa}$ and $\mathcal{I}$ is the ideal of all bounded subsets of $(X, \mathcal{E})$. Since $\left(X,[X]^{<\kappa}\right)$ is free with respect to $\mathfrak{M}_{\kappa}$, the identity mapping $i d:\left(X,[X]^{<\kappa}\right) \longrightarrow(X, \mathcal{E})$ is coarse so $[X]^{<\kappa} \subseteq \mathcal{I}$ and $(X, \mathcal{E}) \in \mathfrak{M}_{\kappa}$.

Remark 2. Every metric $d$ on a set $X$ defines the coarse structure $\mathcal{E}_{d}$ on $X$ with the base $\{(x, y): d(x, y) \leq n\}, n \in \omega$. A coarse structure $\mathcal{E}$ on $X$ is called metrizable if there exists a metric $d$ on $X$ such that $\mathcal{E}=\mathcal{E}_{d}$. By [8, Theorem 2.1.1], $\mathcal{E}$ is metrizable if and only if $\mathcal{E}$ has a countable base. From the coarse point of view, metric spaces are studding in Asymptotic Topology, see [1].

We assume that a variety $\mathfrak{M}$ of coarse space contains an unbounded metric space $(X, d)$ and show that $\mathfrak{M}=\mathfrak{M}_{\omega}$. We choose a countable unbounded subset $Y$ of $X$ and note that $(Y, d) \notin \mathfrak{M}_{\kappa}$ for $\kappa>\omega$ so $(Y, d) \in \mathfrak{M}_{\omega} \backslash \mathfrak{M}_{\kappa}$ and the variety generated by $(X, d)$ is $\mathfrak{M}_{\omega}$.

\section{Comments}

1. Let $G$ be a group with the identity e. An ideal $\mathcal{I}$ in $\mathcal{P}_{G}$ is called a group ideal if $[G]^{<\omega} \subseteq \mathcal{I}$ and $A B^{-1} \in \mathcal{I}$ for all $A, B \in \mathcal{I}$.

Let $X$ be a $G$-space with the action $G \times X \longrightarrow X,(g, x) \longmapsto g x$. We assume that $G$ acts on $X$ transitively, take a group ideal $\mathcal{I}$ on $G$ and consider the coarse structure $\mathcal{E}(G, \mathcal{I}, X)$ on $X$ with the base $\left\{\varepsilon_{A}: A \in \mathcal{I}, e \in A\right\}, \varepsilon_{A}=\{(x, g x): x \in X, g \in A\}$. Then $B\left(x, \varepsilon_{A}\right)=A x, A x=\{g x: g \in A\}$. 
By [4, Theorem 1], for every coarse structure $\mathcal{E}$ on $X$, there exist a group $G$ of permutations of $X$ and a group ideal $\mathcal{I}$ in $\mathcal{P}_{G}$ such that $\mathcal{E}=\mathcal{E}(G, \mathcal{I}, X)$.

Now let $X=G$ and $G$ acts on $X$ by the left shifts $x \longmapsto g x, g \in G$. We denote $(G, \mathcal{E}(G, \mathcal{I}, G))$ by $(G, \mathcal{I})$ and say that $(G, \mathcal{I})$ is a right coarse group. If $\mathcal{I}=[G]^{<\omega}$ then $(G, \mathcal{I})$ is called a finitary right coarse group. In the metric form, these structures on finitely generated groups play an important role in Geometric Group Theory, see [2, Chapter 4].

A group $G$ endowed with a coarse structure $\mathcal{E}$ is a right coarse group if and only if, for every $\varepsilon \in \mathcal{E}$, there exists $\varepsilon^{\prime} \in \mathcal{E}$ such that $(B(x, \varepsilon)) g \subseteq B\left(x g, \varepsilon^{\prime}\right)$ for all $x, g \in G$. For group ideals and coarse structures on groups see [7] or [8, Chapter 6].

2. A class $\mathfrak{M}$ of right coarse groups is called a variety if $\mathfrak{M}$ is closed under formation of subgroups, coarse homomorphic images and products.

Let $\mathcal{K}$ be a class of right coarse groups, $G$ be a group generated by a subset $X \subset G$. We say that a right coarse group $(G, \mathcal{I})$ is free with respect to $\mathcal{K}$ if, for every $\left(G^{\prime}, \mathcal{I}^{\prime}\right) \in \mathcal{K}$, any mapping $X \longrightarrow G^{\prime}$ extends to the coarse homomorphism $(G, \mathcal{I}) \longrightarrow\left(G^{\prime}, \mathcal{I}^{\prime}\right)$. Then Lemma 1, Lemma 2 and Theorem 1 hold for right coarse groups in place of coarse spaces.

Let $\mathfrak{M}$ be a variety of right coarse groups. We take an arbitrary $(G, \mathcal{I}) \in \mathfrak{M}$, delete the coarse structure on $G$ and the class $\mathfrak{M}^{b}$ of groups. If $(G, \mathcal{I}) \in \mathfrak{M}$ then $\left(G, \mathcal{P}_{G}\right) \in \mathfrak{M}$. It follows that $\mathfrak{M}^{b}$ is a variety of groups.

Now let $\mathcal{G}$ be a variety of group different from the variety of singletons. We denote by $\mathcal{G}_{\text {bound }}$ the variety of right coarse groups $\left(G, \mathcal{P}_{G}\right), G \in \mathcal{G}$. For an infinite cardinal $\kappa$, we denote by $\mathcal{G}_{\kappa}$ the variety of all $\kappa$-bounded right coarse groups $(G, \mathcal{I}), G \in \mathcal{G}$.

Let $\mathfrak{M}$ be a variety of right coarse groups such that $\mathfrak{M}^{b}=\mathcal{G}$. Repeating arguments proving Theorem 2, we conclude that $\mathfrak{M}$ lies in the chain

$$
\mathcal{G}_{\text {bound }} \subset \ldots \subset \mathcal{G}_{\kappa} \subset \ldots \subset \mathcal{G}_{\omega} \text {. }
$$

If $G$ is a group of cardinality $\kappa$ and $G \in \mathcal{G}$ then $\left(G,[G]^{<\kappa}\right) \in \mathcal{G}_{\kappa} \backslash \mathcal{G}_{\kappa^{\prime}}$ for each $\kappa^{\prime}>\kappa$. Hence, all inclusions in above chain are strict.

3. let $\Omega$ be a signature, $A$ be an $\Omega$-algebra and $\mathcal{E}$ be a coarse structure on $A$. We say that $A$ is a coarse $\Omega$-algebra if every $n$-ary operation from $\Omega$ is coarse as the mapping $(A, \mathcal{E})^{n} \longrightarrow(A, \mathcal{E})$. We note that each coarse group is a right coarse group but the converse statement needs not to be true, see [8, Section 6.1].

A class $\mathfrak{M}$ of coarse $\Omega$-algebra is called a variety if $\mathfrak{M}$ is closed under formation of subalgebras, coarse homomorphic images and products. Given a variety $\mathfrak{M}$ of coarse algebras, the class $\mathfrak{M}^{b}$ of all $\Omega$-algebras $A$ such that $(A, \mathcal{E}) \in \mathfrak{M}$ is a variety of $\Omega$-algebras.

Let $A$ be a variety of $\Omega$-algebras different from the variety of singletons. We denote by $\mathcal{A}_{\text {bound }}$ the variety of coarse algebras $\left(A, \mathcal{P}_{A}\right), A \in \mathcal{A}$. For an infinite cardinal $\kappa$ we denote by $\mathcal{A}_{\kappa}$ the variety of all $\kappa$-bounded $\Omega$-algebras $(A, \mathcal{E})$ such that $A \in \mathcal{A}$.

If $\mathfrak{M}$ be a variety of coarse algebras such that $\mathfrak{M}^{b}=\mathcal{A}$ then $\mathfrak{M}$ lies in the chain

$$
\mathcal{A}_{\text {bound }} \subseteq \ldots \subseteq \mathcal{A}_{\kappa} \subseteq \ldots \subseteq \mathcal{A}_{\omega},
$$


but, we can not state that all inclusions are strict. In the case of course groups, this is so because each non-trivial variety of groups contains some Abelian group $A$ of cardinality $\kappa$ and the coarse group $\left(A,[A]^{<\kappa)}\right.$ is $\kappa$-bounded but not $\kappa^{+}$-bounded.

4. A class $\mathfrak{M}$ of topological $\Omega$-algebras (with regular topologies) is called a variety (a wide variety) if $\mathfrak{M}$ is closed under formation of closed subalgebras (arbitrary subalgebras), continuous homomorphic images and products. The wide varieties and varieties are characterized syntactically by the limit laws [10] and filters [5]. In our coarse case, the part of filters play the ideals $[X]^{<\kappa}$.

There are only two wide varieties of topological spaces, the variety of singletons and the variety of all topological spaces, but there is a plenty of varieties of topological spaces. The variety of coarse spaces $\mathfrak{M}_{\kappa}$ is a twin of the varieties of topological spaces in which every subset of cardinality $<\kappa$ is compact. We note also that $\mathcal{G}_{\kappa}$ might be considered as a counterpart of the variety $T(\kappa)$ of topological groups from [3], $G \in T(\kappa)$ if and only if each neighborhood of $e$ contains a normal subgroup of index strictly less then $\kappa$.

\section{References}

[1] A. Dranishnikov, Asymptotic topology, Russian Math. Surveys 2000, 55, 1085-1129, DOI: 10.1070/RM2000v055n06ABEH000334. Available online: URL https://arxiv.org/abs/math/9907192

[2] P. Harpe, Topics in Geometrical Group Theory, University Chicago Press, USA, 2000.

[3] S. Morris, P. Nickolas, V. Pestov, Limit laws for wide varieties of topological groups II, Houston J. Math. 2000, 26, 17-27. Available online: URL http://www.sidneymorris.net/Morris123.pdf

[4] O.V. Petrenko, I.V. Protasov, Balleans and G-spaces, Ukr. Math. J. 2012, 64, 387393. Available online: URL http://umj.imath.kiev.ua/archiv/2012/03/0344.pdf

[5] I. Protasov, Varieties of topological algebras, Siberian Math. J. 1984, 25, 783-790. Available online: URL https://link.springer.com/article/10.1007

[6] I. Protasov, T. Banakh, Ball Structures and Colorings of Groups and Graphs, Publisher: Math. Stud. Monogr. Ser., Lviv, 2003, Vol. 11, VNTL.

[7] I.V. Protasov, O.I. Protasova, Sketch of group balleans, Math. Stud. 2004, 22, $10-20$.

[8] I. Protasov, M. Zarichnyi, General Asymptology, Publisher: Math. Stud. Monogr. Ser., Lviv, 2007, pp. 219, Vol. 12, VNTL. 
[9] J. Roe, Lectures on Coarse Geometry, AMS University Lecture Ser., Providence, R.I., 2003, pp. 176, Vol. 31.

[10] W. Taylor, Varieties of topological algebras, J. Austral. Math. Soc. 1977, 23, 207-241.

\section{CONTACT INFORMATION}

I. Protasov:

Faculty of Computer Science and Cybernetics

Kyiv University

Academic Glushkov pr. 4d

03680 Kyiv, Ukraine

i.v.protasov@gmail.com 